\title{
A Research of Airfield Lamp Monitoring System Based on WSNs WANG Bingyuan ${ }^{\mathrm{a}}, \mathrm{XU}$ Minghua $^{\mathrm{b}}$ \\ Aeronautical Automation College, Civil Aviation University of China, Tianjin, 300300, China \\ awangbingyuan@eyou.com, Isyshuihuo@126.com
}

Keywords: single lamp monitoring; wireless sensor networks; low power consumption; routing protocol

\begin{abstract}
To improve the reliability of airfield lighting, a new method based on wireless sensor networks is put forward. As the energy consumption of the sensor node determines the lifetime of the WSNs, the key problem of WSNs is designing a low-energy sensor node and adopting low-energy routing protocol. According to the location of the lamps, a new routing protocol on the base of clustering network with periodic sleep-wake up mechanism is approached. The initial tests show that the system can collect the lamps' information periodically and meet the requirements of real time monitoring.
\end{abstract}

\section{Introduction}

In low visibility and other complicated weather conditions, airfield lighting system is an important visual auxiliary navigation equipment to guarantee the plane's safe taking off, landing and sliding in the night. Its working condition and reliability is crucial to the safety of the plane. Failure of lights may lead to accidents, so it is necessary to monitor lamp's working conditions ${ }^{[1]}$.

The earliest lights monitoring systems mostly adopt the power line carrier technology. But there are still some problems, such as, the reliability of electric power carrier signal modulation circuit, modulation parameters optimization and signal demodulation algorithm need to be solved. Furthermore, the monitoring unit may short-circuit the lights when it is modulating the information to the power supplication circuit. The perceptual load and large numbers of harmonic components in non sine wave power line make the data transmission and demodulation algorithm more difficult.

In new airports, this information can be sent by optical fiber which is buried in advance when constructing the airport, but it does not apply to the current operating airports with no optical fiber.

Nowadays wireless sensor network (wireless sensor networks, WSNs) has been widely applied to environmental monitoring systems and a variety of sensor nodes have been developed by research institutions ${ }^{[2-3]}$. Adopting WSNs based single-lamp monitoring system can effectively eliminate the problem caused by power line carrier technology.

\section{System Composing Scheme}

In WSNs monitoring system, each monitoring node has two functions: gathering information periodically and sending the data to monitoring center by WSNs. Five parts are included in the system, as shown in the figure 1 . The monitoring center is too far away from the monitoring area to communicate with the wireless base station directly so the single-lamp monitoring system takes the layered network. The information collected by the sensor nodes transmit through head-cluster, sink node and base station, and finally get to the monitoring center.

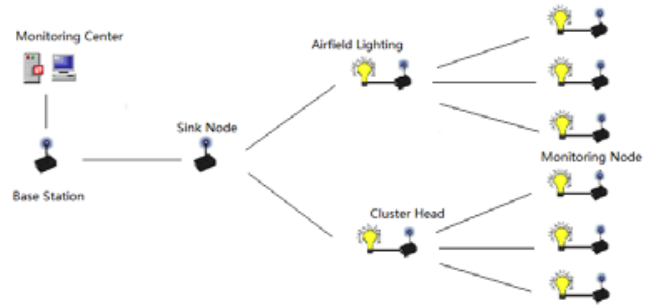

Figure 1. Structure of the monitoring system 
A new round of inspection mission begins with the sink node receiving the inspection instruction that sent by the monitoring center. Then nodes collect the information of the lamp and send it to the cluster. The sink nodes receive all the data from the cluster head node. The inspection mission ends when the data is sent to the base station. The WSNs accomplish the inspection mission.

\section{Hardware Design}

Sensor node is the basis of the system and it consists of MCU module, radio transceiver module, data acquisition module and power module as shown in figure $2^{[4]}$. Microcontroller manages the sensor node and allocates resources. Radio transceiver module exchanges data between each other. Data acquisition module samples voltage on lamps. Power module supports node to operate normally.

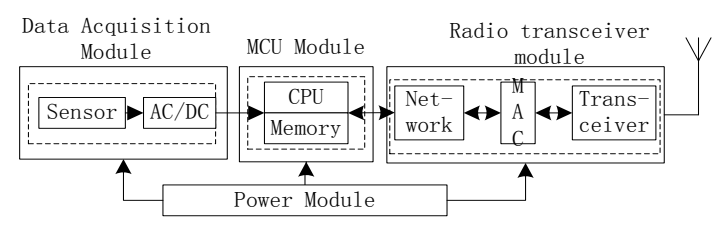

Figure 2. The structure of sensor node

According to the ICAO ANNEX 14, the additionally installed monitoring instruments cannot affect the lighting system's normal working even when additional monitoring instruments fail ${ }^{[5]}$. The system data acquisition module is isolated from the lamp circuit. At the same time, batteries are used as the power to improve reliability. Nodes consume energy once they are working while the energy of battery is limited. To prolong the lifetime of the network it's necessary to design low-power hardware and software.

\section{A. MCU Module}

In this system MSP430G2553 is selected as the microcontroller module, which is an ultra-low energy chip and can work normally when powered between $1.8 \mathrm{~V}$ and 3.6V. The MSP430G2553 has one active mode and five software selectable low-power modes of operation, in which the power consumption is different. For example, the shutdown mode consumption is 0.1ìA much less than 2.6ìA in active mode. This microcontroller is embedded with an ADC module, two comparators and many other peripheral units. It fully meets the requirement of the node's operation.

\section{B. Radio Transceiver Module}

CC1101 is a low-power radio chip employed as radio transceiver. It consumes only 0.2ìA in idle mode and 14mA in receive mode. CC1101 have powerful functions including data packet automatically assembling or disassembling, idle channel evaluation, wireless waking, CRC verification and so on.

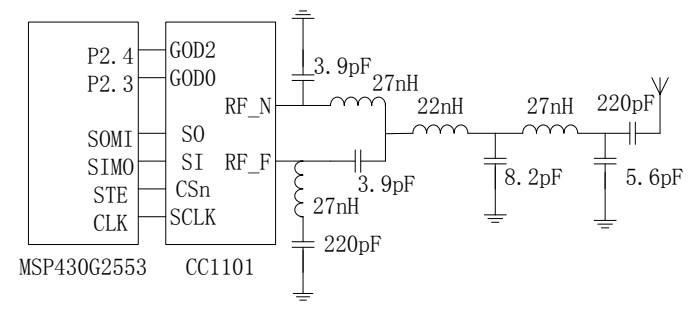

Figure 3. CC1101 Connects with microcontroller

The circuit network that matches the antenna is shown in figure $3^{[6]}$. Here impedance matching is needed for communicating successfully. Antenna is whip shaped. The relationship between length and frequency is as shown in equation $1^{[7]}$.

$$
L=(c / f) * 0.96 / 4
$$

Where c stands for light speed, $\mathrm{f}$ stands for frequency, 0.96 is wavelength shortening rate, $\mathrm{L}$ is length of antenna. 
When node is working at $433 \mathrm{MHz}$, the wavelength is $66.5 \mathrm{~cm} .1 / 4$ wavelength antenna is $16.6 \mathrm{~cm}$.

\section{Data Acquisition Module}

The airfield lighting system is powered by a constant current. Lamps are connected in series in the loop by an isolation transformer that ensures all the lamps are on the same light intensity determined by the current size. The current can be adjusted by controlling SCR conduction angle. There are five adjustable current levels (2.8A, 3.4A, 4.1A, 5.2A and 6.6A) when the airfield lighting system works normally $^{[1]}$. Workers can choose one of them depending on the weather condition. The working state of the light can be evaluated by sampling voltage on it. The structure of voltage acquisition module is shown in figure 4.

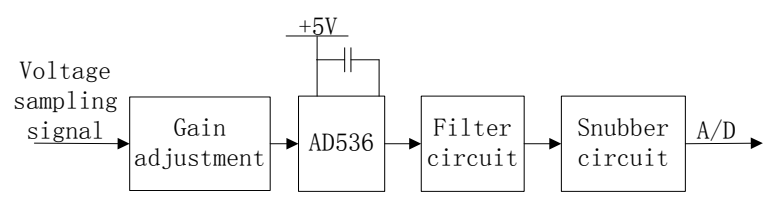

Figure 4. The structure of voltage acquisition module

A voltage is sampled and sent to AD536. The function of AD536 is transforming the AC signal to a DC signal. Then the MCU samples DC signal and use it to judge the working state of the lamp.

D. Power Module

Two batteries are used to supply power. A voltage transformer is applied to transform the power of the two batteries to $3 \mathrm{~V}$ and $5 \mathrm{~V}$. In an running system, once the MCU detects the batteries energy is lower than a level, information would be sent to the monitoring center.

\section{Software Design}

In the system the nodes exchange information through the Airfield Clustering Algorithm based on Distance and Energy (AL-CADE) ${ }^{[8]}$. In the protocol, the monitoring area was set several small logic zones according to geography locations of the nodes, and there were some advanced nodes placed in the logic zones as relay nodes. The cluster-head number of the nodes was optimized in the elliptical area, and the cluster-head election algorithm based on energy and distribution of nodes was put forward. If the nodes in a cluster having different amount of energy at the same time and being located in a place with a small distant to other nodes, them the node with the highest energy should be cluster-head to ensure that the energy consumption is evenly distributed among all the nodes and all nodes die at approximately the same time.

$$
T^{\prime}(n)=\left\{\begin{array}{cc}
\frac{c_{\text {opt }}}{N-c_{\text {opt }}\left[r \bmod \left(\frac{N}{c_{\text {opt }}}\right)\right]} \times \frac{E_{\text {ori }}-t E_{\text {ove }}^{r}}{E_{\text {ori }}-t E_{\text {res }}(i)} \times \frac{d_{\text {ove }}}{d(i)} & n \in G \\
0 & \text { otherwise }
\end{array}\right.
$$

Where $r$ is the current round, $E_{\text {ori }}$ is the initial energy of a node, $E_{\text {res }}(i)$ is the current energy of node $\mathrm{i}, \mathrm{t}$ is a constant, $\mathrm{d}_{\mathrm{ave}}$ is the avergy distance from the nodes to the base station, $\mathrm{d}(\mathrm{i})$ is the distance from node $i$ to the base station

The cluster head elected then broadcast in its own logic zone, and the non-cluster head nodes sent request to the nearest cluster head for joining in. After each node has decided to which cluster it belongs, the TDMA schedule based on the address of the nodes in the cluster is fixed and broadcast back to the nodes telling each node when it can transmit. As shown in figure 5.

The cluster head node would judge whether there is monitoring node is fail to transmit data, if so it will require the monitoring node upload data again. Until all the data from the non-cluster heads nodes has been received, all the information will be sent to relay node in its logic zone.

In order to save energy, all the nodes are in sleep mode after inspection and wait for the next inspection mission's coming. If detecting circuit detects fault of the light, the node will wake up from sleeping mode and report the failure to the cluster head node immediately. 


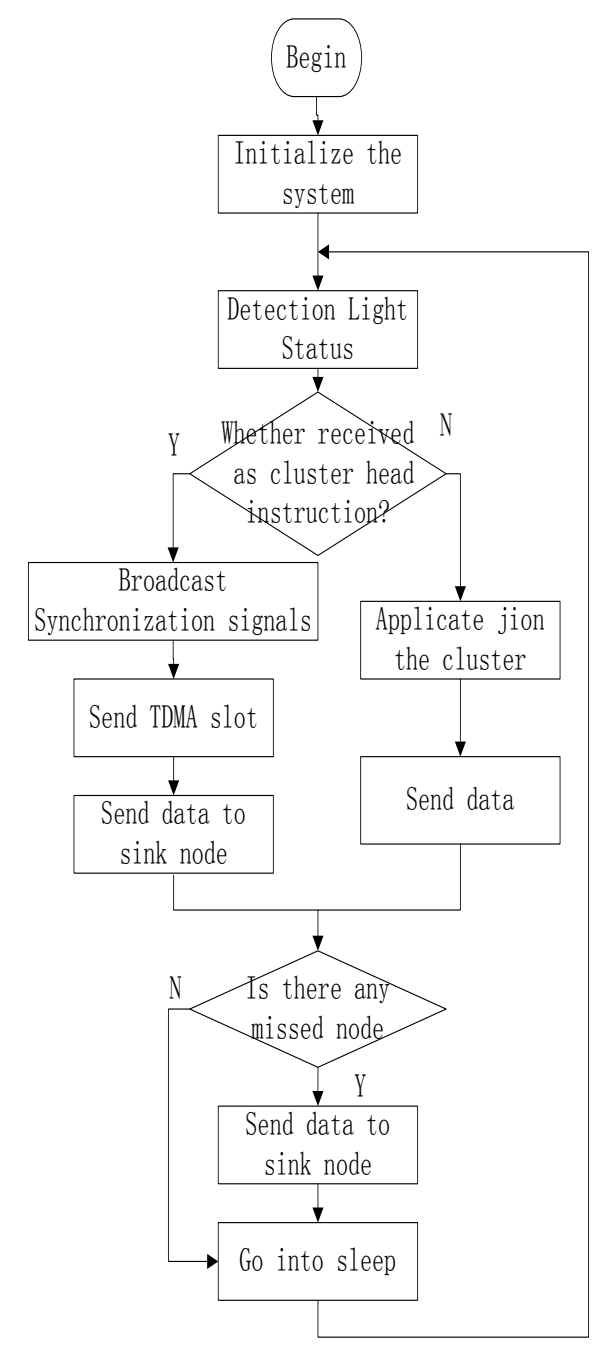

Figure 5. Working flow chart of the node

\section{Experiment and Analysis}

To verify the reliability of the sensor nodes, a series of experiments were finished. Five nodes are used and three of them were used as common monitoring nodes, one was the sink node, the other one was regarded as the base station who can communicate with the computer through USB interface. The interface of the monitor system is developed by using Visual Basic 6.0. The database is built by using Access 2000. The analysis of the experiment focuses on the relationship between the distance and the node's reliability. As shown in the experiments, when distance increased, the rate of receiving error data and missing data increased as well. The data can be transmitted to100m away on the speed of $3.2 \mathrm{Kbps}$ and that meets the need of airfield lighting monitoring system.

In order to estimate the node's lifetime, communication experiment tests were finished. The distance between two nodes was greater than $25 \mathrm{~m}$, and they exchanged data once every minute until one of them ran out of energy. The result is shown in Table 1 ,

Table 1. Tests Result

\begin{tabular}{|l|l|l|l|l|l|}
\hline Test No. & 1 & 2 & 3 & 4 & 5 \\
\hline Patrolling times & 353 & 362 & 349 & 364 & 361 \\
\hline
\end{tabular}

To guarantee the reliability of the airfield lighting, it requires an inspection of all the lights in a day. Double batteries can support inspection for 360 rounds, which means the node can continue working for more than one year ignoring the energy consumption on sleep and emergency state. 


\section{Conclusion}

The application of WSNs to the single-lamp monitoring system can improve the reliability of navigation lighting system and the security of the airplane approaching and landing. The primary test results show that the system can be used to monitor the work state of the lamps timely and reliably. The system with low-power chip and effective energy-saving control algorithm can prolong the network lifetime effectively.

\section{Acknowledgement}

This Work is Sponsored by the National Natural Science Foundation U1333102.

\section{Reference}

[1] Gao Shu-ling, Wang Yun-ling. airport visual aids [M] Tianjin: Tianjin Science and Technology Press, 2000.98-100

[2] Long Hui. Research on development of wireless sensor network status [J] Microcontroller and Embedded Systems, 2011 (6): 9-12

[3] Si Hai-fei, Yang Zhong, Wang Jun. Review on research status and application of wireless sensor networks[J]. Mechanical and Electrical Engineering, 2011 (1): 16-20 + 37.

[4] Zhang Yong-mei, Yang Chong, Ma Li, Wang Kai-feng. Design Method of Wireless Sensor Network Node with Low Power Consumption[J]. Computer Engineering, 2012 (3): 71-73

[5] ICAO Annex XIV - Airport [S] 20-27.

[6] Ding Xin, Xu Bu-gong. Street Light Monitoring and Energy-saving System Based On CC1101 Wireless Ad Hoc Network[J]. Automation and Instrumentation, 2011 (2): 20-23 + 49.

[7] Richard Chi-Hsi Li. Key Issues In RF/RFIC Circuit Design[M]. Translated by Wang Zhi-gong .Beijing: Higher Education Press, 2007.

[8] Jiang Yang, Sun Liulin, Ao Wenjun, Yuan Min Research on optimal cluster-head number of LEACH routing protocol for WSN [J]. Application Research of Computers, 2010,11: 4251-4253. 